\section{Kinderaugen benötigen Kompetenz und Geduld}

Mit diesen und ähnlichen Werbesprüchen haben die Optiker in den vergangenen Wochen für sich als kompetente Partner geworben. Optiker halten Vorträge vor Lehrern und werben für ihre Zusammenarbeit. Die Schulen schicken verhaltensauffällige Kinder in gutem Glauben zu bestimmten ,qualifizierten" Optikern oder in sogenannte Lerninstitute wie das BEIKA-Institut.

Um dieser Entwicklung entgegenzuwirken, hat die Fachgruppe Augenheilkunde und Optometrie der Ärztekammer für Wien Aufklärungsarbeit in Form einer Pressekonferenz betrieben, einen Fortbildungsvortrag angeboten und die Teilnehmer dieser Fortbildung auf einer Liste als Ansprechpartner für Kinder mit Teilleistungsstörungen veröffentlicht.

Nach ausgiebiger Diskussion vertreten wir nun die Auffassung, dass allen österreichischen Augenärzten Fortbildungsmöglichkeiten auf dem Gebiet der kindlichen Ophthalmologie geboten werden sollen. Dieses Fortbildungsangebot soll den Bogen von der Wiederholung gezielter Untersuchungsmethoden bis hin zum Legasthenietraining spannen. Die Fortbildung soll geteilt in einem ophthalmologischorthoptischen und einen psychologischen Teil erfolgen. Der Vorschlag für die Leistungsbezeichnung im kassenfreien Raum lautet: „Untersuchungsmodul für okuläre Lesestörung und binokuläre Dysfunktion bei Verdacht auf Teilleistungsschwäche“. Mit dem nachweislichen Besuch dieser nachstehenden Fortbildungseinheiten soll der Erwerb eines diesbezüglichen Fortbildungs-Diploms der ÖOG verbunden sein. Diese Themen werden auch wiederholt angeboten werden. Ebenso zählen sie zum DFP der Ärztekammer.

Zur Zeit stehen folgende Termine für diese Fortbildungseinheiten im Großen Sitzungssaal der Ärztekammer für Wien, 1010 Wien, Weihburggasse 10-12, fest:

Freitag, 13. Jänner 2006, 18.00 Uhr: 1. Teilveranstaltung:

Thema: Teilleistungsschwächen und ihre Auswirkungen in Legasthenie, Dyskalkulie und anderen Lernstörungen im Kindesalter - eine Schnittstelle zwischen Ophthalmologie und klinischer Psychologie.

Dr. Brigitte Sindelar, klin. Psychologin, Kosten: 60 Euro.

Freitag, 27. Jänner 2006, 18.00 Uhr: 2. Teilveranstaltung:

Thema: Ophthalmologische orthoptische Aspekte in der Diagnose und Therapie der okulären Lese-RechtschreibStörung.

Prof. Dr. Andrea Langmann, Univ.-Augenklinik Graz, Kosten: 60 Euro.

Praxis: Im Anschluss an die theoretische Modulveranstaltung werden praktische Workshops in verschiedenen Schielabteilungen Österreichs angeboten.
Suche dieses Bild!

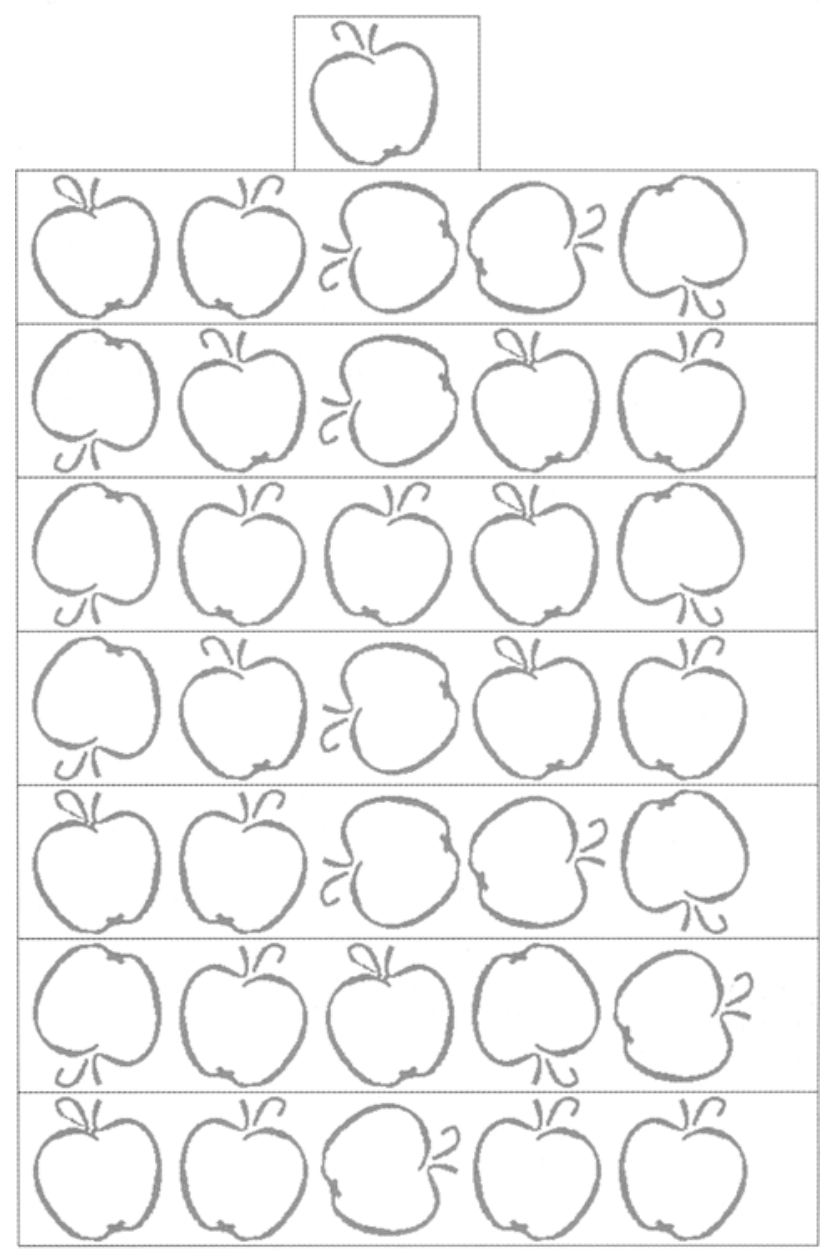

Peggy Sippel 2001 www.legasthenie-trainer.de

Dienstag, 21. Februar 2006: Thema folgt.

Dienstag, 14. März 2006: Thema folgt.

Wir hoffen, dass zahlreiche Kollegen von diesem Angebot Gebrauch machen und als Inhaber dieses Diploms dann vermehrt von hilfesuchenden Eltern und Lehrern angesprochen werden.

Prim. Prof, Dr. Paul Drobec

Vorsitzender der Kommission für Refraktion und Optometrie der ÖOG 